\title{
Prevenção e intervenção à dependência de álcool ao idoso na Atenção Primária à Saúde: Revisão integrativa da literatura
}

\author{
José Stéfano Faia Destro1, Elza de Fátima Ribeiro Higa1, Márcia \\ Aparecida Padovan Otani' ${ }^{1}$, Maria José Sanches Marin'1, Rodolfo de \\ Oliveira Medeiros $^{1}$ Jaqueline Dias do Nascimento ${ }^{2}$ \\ ${ }^{1}$ Faculdade de Medicina de Marília, Estado de São Paulo, Brasil | fandestro@hotmail.com; \\ hirifael@gmail.com; mpadovanotani@gmail.com; marnadia@terra.com.br; \\ rodorfo.famema@hotmail.com | https://orcid.org/0000-0003-1776-3584; \\ https://orcid.org/0000-0001-5772-9597; https://orcid.org/0000-0001-9540-4996; \\ https://orcid.org/0000-0001-6210-6941; https://orcid.org/0000-0002-4930-684X \\ 2 Universidade Federal do Paraná, Estado do Paraná, Brasil | jaquelineddias1@hotmail.com \\ | https://orcid.org/0000-0002-4249-4809
}

Resumo: Introdução: $O$ Brasil tem vivenciado um rápido processo de transição demográfica trazendo consigo novos desafios à saúde pública, dentre eles, a dependência de álcool entre idosos. Objetivo: analisar as evidências literárias sobre as ações de prevenção e intervenção para ao idoso dependente de álcool na Atenção Primária à Saúde (APS). Método: Revisão Integrativa da Literatura realizada em 2020 nas bases de dados LILACS, BDEnf, CUMED, IBECS, MedLine, SciELO, SCOPUS e Web of Science, a partir dos seguintes descritores: "Idoso", "Álcool", "Alcoolismo", "Transtornos Relacionados ao Uso de Álcool" e "Atenção Primária à Saúde". Os critérios de inclusão foram: artigos originais disponíveis on-line na íntegra, nos idiomas inglês, português e espanhol e os de exclusão artigos de revisão bibliográfica, teses, dissertações e opinião de especialistas. Foram identificados um total de 1159 artigos, após exclusão por duplicidade, aplicação de critérios de exclusão e análise qualitativa, permaneceram seis artigos. Resultados: da análise dos dados obtidos foram estruturadas três categorias: Necessidade de triagem para consumo de álcool em pacientes idosos da APS, Eficácia de Intervenção Breve para consumo de álcool na APS e Barreiras para implementação de triagem para consumo de álcool e Intervenção Breve. Conclusões: a aplicação de estratégias de enfrentamento ao consumo de álcool aos pacientes idosos da APS possui custo benefício comprovado, porém não são amplamente realizadas, havendo mais barreiras do que facilitadores para sua implementação. Diante deste contexto, é necessário a realização de estudos objetivando avaliar a eficácia da triagem para consumo de álcool e Intervenção Breve em idosos devido a escassez de estudos em âmbito nacional.

Palavras-chave: Idoso; Álcool; Alcoolismo; Transtornos Relacioandos ao Uso de Álcool; Atenção Primária à Saúde.

Prevention and Intervention of Alcohol Dependence to the Elderly in Primary Health Care: An Integrative Literature Review

Abstract. Introduction: Brazil has experienced a rapid demographic transition process, bringing with it new challenges to public health, among them, alcohol dependence among the elderly. Objective: to analyze the literary evidence on prevention and intervention actions for the elderly dependent on alcohol in Primary Health Care (PHC). Method: Integrative Literature Review carried out in 2020 in the LILACS, BDEnf, CUMED, IBECS, MedLine, SciELO, SCOPUS and Web of Science databases, based on the following descriptors: "Elderly", "Alcohol", "Alcoholism", "Disorders Related to Alcohol Use" and "Primary Health Care". Inclusion criteria were: original articles available online in full, in English, Portuguese and Spanish, and exclusion articles for bibliographic review, theses, dissertations and expert opinion. A total of 1159 articles were identified, after exclusion due to duplication, application of exclusion criteria and qualitative analysis, six articles remained. Results: From the analysis of the data obtained, three categories were structured: Need for screening for alcohol consumption in elderly patients in PHC, Efficiency of Brief Intervention for in elderly patients in PHC and Barriers for implementing screening for alcohol consumption. alcohol and Brief Intervention. Conclusions: the application of strategies to cope with alcohol consumption to elderly patients in Primary Health Care has proven cost benefit, but they are not widely implemented, with more barriers than facilitators for their implementation. In this context, it is necessary to carry out studies aimed at evaluating the effectiveness of screening for alcohol consumption and Brief Intervention in the elderly due to the scarcity of studies nationwide.

Keywords: Elderly; Alcohol; Alcoholism; Disorders Related to Alcohol Use; Primary Health Care. 


\section{Introdução}

O Brasil tem vivenciado um rápido processo de transição demográfica trazendo consigo novos desafios à saúde pública, dentre eles, a dependência de álcool, que está associada a indivíduos mais jovens, porém dados indicam que este quadro vem aumentando entre idosos simultaneamente ao crescimento dessa população, sendo cada vez mais reconhecido com um problema de saúde pública (Chagas et al., 2019).

Acontecimentos no decorrer da vida dos idosos como aposentadoria, desemprego, perda de familiares e amigos, solidão e isolamento social, podem deixá-los vulneráveis a adoção de hábitos nocivos, como o consumo abusivo de álcool (Adayonto et al., 2019; Chagas et al., 2019).

Devido a alterações inerentes ao processo de envelhecimento, os malefícios à saúde física, mental e social decorrentes da dependência de álcool são piores nos idosos, embora seus casos sejam pouco identificados sendo imperativo a implementação de ações de enfrentamento à essa problemática. O Programa do Ministério da Saúde para Atenção Integral a Usuários de Álcool e Outras Drogas estabelece que a assistência a usuários de álcool deve ser oferecida em todos os níveis de atenção, privilegiando os cuidados em dispositivos extra-hospitalares, devendo estar inserida na atuação do Programa de Saúde da Família (Adayonto et al., 2019; Abreu et al., 2017; Brasil, 2003).

Neste cenário, os serviços da Atenção Primária à Saúde (APS), se apresentam como de suma importância para aplicação de estratégias de enfrentamento ao consumo abusivo de álcool. A utilização de técnicas terapêuticas concisas e de curta duração na APS como triagem para consumo de álcool e Intervenção Breve (IB) possuem benefícios comprovados na população idosa, facilidade de execução e redução de gastos em serviços de saúde, porém apresentam barreiras para sua implementação (Abreu et al., 2017; Finn et al., 2018; Pereira et al., 2013).

Nesse contexto, este estudo tem como objetivo analisar as evidências literárias sobre as ações de prevenção e intervenção para o idoso dependente de álcool nos serviços de APS.

\section{Método}

Trata-se de uma Revisão Integrativa da Literatura, (RIL), método desenvolvido em seis etapas: 1) identificação do tema e seleção da hipótese ou questão de pesquisa; 2) estabelecimento de critérios de inclusão e exclusão de estudos; 3) definição das informações a serem extraídas dos estudos selecionados/categorização dos estudos; 4) avaliação dos estudos incluídos na revisão integrativa; 5) interpretação dos resultados e 6) apresentação da revisão/síntese do conhecimento (Souza et al., 2010).

Para a elaboração da pergunta de pesquisa, este estudo seguiu as recomendações do Joanna Briggs Institute para pesquisas qualitativas, utilizando-se da estratégia PICo, acrônimo para P: População; I: Interesse; Co: Contexto (Briggs, 2014). Assim, a questão norteadora foi: Quais as ações de prevenção e intervenção à dependência de álcool ao idoso na Atenção Primária à Saúde? A estratégia PICo foi empregada da seguinte forma: P: Idosos dependentes de álcool; I: Prevenção e Intervenção; Co: Atenção primária à saúde. A busca dos estudos ocorreu em fevereiro de 2020 e contou com os seguintes descritores selecionados nos Descritores em Ciência da Saúde (DeCS): "Idoso", "Álcool", "Alcoolismo", "Transtornos Relacionados ao Uso de Álcool", "Atenção Primária à Saúde".

As bases de dados utilizadas foram: Literatura Latino-Americana e do Caribe em Ciências da Saúde (LILACS), Base de dados em Enfermagem (BDEnf), Centro Nacional de Informações de Ciências Médicas de Cuba (CUMED), Índice Bibliográfico Espanhol em Ciências da Saúde (IBECS), Index Psi, Medical Literature Analisys and Retrievel System Online (MedLine), Scientific Eletronic Library Online (SciELO), SCOPUS e Web of Science. 
Os critérios de inclusão foram: artigos originais disponíveis on-line na íntegra, nos idiomas inglês, português e espanhol e de exclusão: artigos de revisão bibliográfica, teses e dissertações e opinião de especialistas.

A amostra inicial identificada nas bases de dados foi de 1159 estudos, após exclusão dos estudos duplicados, 1123 estudos foram selecionados para leitura dos títulos, sendo 179 selecionados para leitura do resumo. Em seguida, foram selecionados 54 estudos para leitura na íntegra, sendo removidos 29 estudos após aplicação dos critérios de exclusão, restando 29 estudos incluídos na análise qualitativa. Permaneceram na amostra final seis estudos que atenderam aos critérios de inclusão e responderam à pergunta de pesquisa. O Fluxograma 1 ilustra o processo de seleção dos estudos:

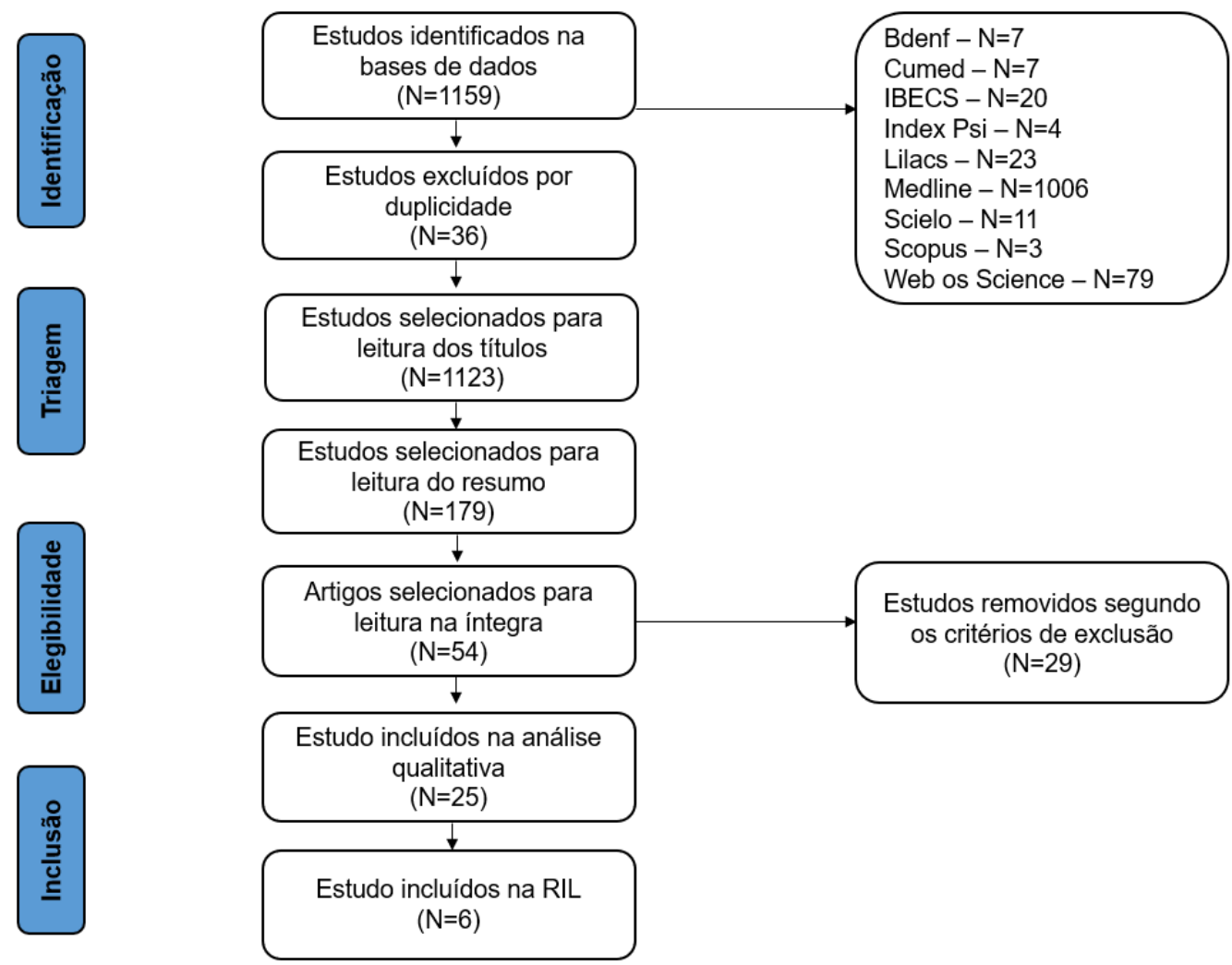

Fluxograma 1. Seleção amostral das publicações sobre a prevenção e intervenção à dependência de álcool ao idoso na APS.

Adaptado de Moher et al., (2009).

A apresentação dos dados obtidos foi desenvolvida de modo descritivo, fundamentada na análise de conteúdo. A análise qualitativa foi inicialmente foi realizada com a leitura flutuante dos artigos selecionados objetivando identificar elementos relevantes nos textos. Posteriormente procedeu-se a exploração do material observando temas que se repetiam nos artigos e escolhendo categorias iniciais, conforme preconizado em literatura referente a RIL (Souza et al., 2010).

\section{Resultados e discussão}

Os artigos selecionados foram publicados entre os anos de 2003 a 2016, houve baixo número de pesquisas que relacionam o consumo abusivo de álcool entre idosos e ações e intervenções a serem realizadas na APS. Os dados dos artigos selecionados foram compilados em dois quadros representativos: 
Quadro 1. Artigos incluídos na revisão integrativa constituído por: título do artigo, autores, ano da publicação, periódico, país de origem, base de dados, tipo de estudo, nível de evidência, participantes e objetivos.

Título do artigo/Autores/ Ano
da publicação/Periódico/ País
de origem/ Base de dados

Consumo de álcool e qualidade de vida em idosos na saúde da família.

Soares, M. S., et al.

Rev. enferm. Cent.-Oeste Min. 2016.

Brasil.

LILACS.

Padrões do uso de álcool em uma amostra de idosos no município de Porto Alegre, Brasil. Guidolin, B. L., et al.

Ciênc. saúde coletiva.

2016.

Brasil.

Web of Science.

A Qualitative Study of Service Provision for

Alcohol Related Health Issues in

Mid to Later.

Haighton, C., et al.

PLoS One.

2016.

Inglaterra.

MEDLINE.

An evaluation of an intervention to assist primary care physicians in screening and educating older patients who use alcohol

Fink, A., et al.

2005

J Am Geriatr Soc.

Estados Unidos da América.

MEDLINE.

Why do older unhealthy drinkers decide to make changes or not in their alcohol consumption? Data from the Healthy Living as You Age Study

Borok, J., et al.

2013.

J Am Geriatr Soc

Estados Unidos da América.

Scopus.

Health Care Utilization by Older Alcohol-Using Veterans: Effects of a Brief Intervention to Reduce At-Risk Drinking.

Copeland, L. A., et al.

2003.

Health Education \& Behavior. 2003.

Estados Unidos da América. MEDLINE

Tipo de estudo
Participantes
Nível de evidência

Tipo: Estudo transversal. Local: Belo Horizonte MG, Brasil.

Nível de evidência: 4.

Participantes: $\quad 593$

idosos.

Nível de evidência: 4.

Tipo: Estudo transversal Local: Porto Alegre - RS, Brasil.

Participantes:

\section{7}

idosos.

Nível de evidência: 4

Avaliar a associação entre o consumo de álcool e qualidade de vida em idosos atendidos na APS de uma metrópole.

Determinar o padrão de uso de álcool e sua associação com características sociodemográficas em uma amostra de pacientes idosos matriculados na APS na cidade de Porto Alegre, Rio Grande do Sul, Brasil

Tipo: Estudo qualitativo. Local: Área urbana em North East England, Inglaterra.

Participantes: 51 adultos com 50 anos ou mais.

Nível de evidência: 4

Tipo: Estudo comparativo prospectivo.

Local: Três centros de atenção primária.

Participantes: 665 idosos e 23 médicos.

Nível de evidência: 4.

Tipo: Análise secundária de dados de um estudo controlado randomizado.

Local: Três locais de atendimento primário.

Participantes: 631

adultos com 55 anos ou mais com consumo de risco de álcool.

Nível de evidência: 4

Tipo: Ensaio clínico randomizado.

Local: Setor privado e clínicas de veteranos na atenção primária.

Participantes: Adultos com 55 anos ou mais identificados como bebedores de risco.

Nível de evidência: 3
Obter compreensão das experiências e atitudes relacionadas ao apoio a problemas de saúde relacionados ao álcool no meio da vida.

Avaliar se os médicos ao educarem pacientes idosos consumidores de álcool de risco com relatos personalizados conseguem reduzir o consumo de álcool.

Entender as características e motivos pelos quais bebedores mais velhos decidem alterar ou manter o consumo de álcool.

Estimar o efeito do uso de uma IB de redução de álcool nos cuidados de saúde 
Quadro 2. Síntese dos principais resultados e conclusões dos artigos selecionados.

Principais resultados

Identificado considerável percentual de idosos em consumo de alto risco e dependência alcoólica, nenhum dos participantes considerou ser alcoolista ou referiram o alcoolismo como um problema de saúde. O estudo aponta que o consumo abusivo de álcool entre idosos implica em menores indicadores de qualidade de vida e satisfação com a saúde.

$12 \%$ dos participantes tinham história de alcoolismo, 9\% foram dependentes de álcool no passado, $3.1 \% \mathrm{com}$ atual diagnóstico de alcoolismo e 2,9\% estão atualmente com problema de abuso de álcool.

As razões para o atraso na busca de ajuda dos dependentes de álcool mais velhos estão relacionadas ao forte estigma associado a ser um consumidor abusivo de álcool e eles desconheciam se havia ou ajuda disponível. Os entrevistados indicaram que os clínicos gerais foram a primeira fonte de ajuda encontrada, porém em muitos casos não os acolheu bem, encaminhando-os para outros serviços ou tentando medicá-los sem ao menos ouvi-los.

Constatado que fornecer aos pacientes idosos de cuidados primários relatórios personalizados de sua classificação de consumo de álcool, juntamente com informações educacionais, houve redução do consumo nocivo em $23 \%$ por um período de 12 meses.

A maioria dos idosos diminuíram o consumo de álcool ao reconhecerem que seu uso podia estar causando danos a sua saúde. Muitos participantes não fizeram alterações porque não acharam que a quantidade de álcool consumida era um problema.

O efeito da utilização da IB nesta amostra de idosos que são bebedores de risco, aumentou o uso de cuidados médicos ambulatoriais a curto prazo, indicando que idoso com atitudes positivas em relação a um comportamento de saúde têm maior probabilidade de manter atitudes positivas em relação a outros comportamentos de saúde. A breve intervenção pode levar a reduções a longo prazo dos cuidados de saúde.

\section{Conclusões}

Maior necessidade de desenvolvimento de estratégias de enfrentamento e políticas públicas para o alcoolismo em faixas etárias mais avançadas, problematizar, divulgar e desmitificar o alcoolismo na velhice, incentivar o diagnóstico precoce e proporcionar alternativas de ajuda na APPS

Necessidade de medidas para combate ao alcoolismo entre idosos, como a utilização de instrumentos de triagem para consumo de álcool e a implementação de IB.

A APS emergiu como importante na identificação de problemas e aconselhamento, sendo o serviço que as pessoas mais velhas primeiramente procuraram ajuda para problemas relacionados ao consumo de álcool. Necessidade de maior treinamento das equipes da APS para identificar e acolher idosos com problemas de consumo de álcool, e não somente encaminhá-los para serviços especializados ou medicá-los.

O estudo reforça que pacientes idosos com problemas de uso de álcool, ao receberem aconselhamentos personalizados de médicos da APS, apresentam grande chance de reduzir o consumo de álcool nocivo à saúde.

O estudo aponta que o aconselhamento na APS é eficaz para redução do consumo de álcool para idosos, conscientizando-os acerca dos malefícios que o consumo abusivo de álcool possa trazer.

Importância da implementação de IB em idosos com problemas de consumo de álcool, leva a uma maior conscientização para redução ou parada total do consumo de álcool, além de se preocuparem com outros aspectos importantes sobre sua saúde.

\section{Fonte: Elaboração própria}

Dos seis artigos selecionados, um foi publicado em 2003, um em 2005, um em 2013, três em 2016 e um em 2018. Quatro estudos foram desenvolvidos nos Estados Unidos da América, três no Brasil e um na Inglaterra. Três estudos possuem delineamento transversal, um estudo com abordagem qualitativa, um estudo comparativo prospectivo, um estudo descritivo de análise secundária de um estudo controlado randomizado e um ensaio clínico randomizado. A maioria dos estudos encontram-se no Nível de Evidência grau 4, conforme preconizado na literatura (Souza et al., 2010). Esta revisão não tem como objetivo discutir os níveis de evidência encontrados. 
A partir da análise de conteúdo, nesta revisão foram estruturadas três categorias analíticas: Necessidade de triagem para consumo de álcool em pacientes idosos da Atenção Primária à Saúde, Eficácia de Intervenção Breve para consumo de álcool na Atenção Primária à Saúde e Barreiras para implementação de triagem para consumo de álcool e Intervenção Breve.

\subsection{Necessidade da Triagem para Consumo de Álcool em Pacientes Idosos da Atenção Primária à Saúde}

A triagem para consumo de álcool objetiva investigar a quantidade, frequência $e$ comportamentos problemáticos decorrentes do consumo de álcool. Poucos sistemas de saúde conseguem implementar com sucesso a triagem para o consumo de álcool, e geralmente não são mantidas ao longo do tempo. Frequentemente, idosos não veem o consumo abusivo de álcool como uma doença, podendo negar seu consumo, mesmo quando Ihes é assegurado a confidencialidade da informação. Desse modo, profissionais da saúde devem atentar-se para a possibilidade de quadro de consumo abusivo de álcool nessa faixa etária (Fink et al., 2005; Guidolin et al., 2016; Shaker \& Ardnt, 2017; Soares et al., 2016).

Baseado em critérios de benefício clínico e custo efetividade, a triagem para consumo de álcool está entre os serviços preventivos mais indicados, porém é pouco realizado (Fink et al., 2005). Shaker and Ardnt (2017) afirmam que todos os pacientes adultos da APS devem passar por triagem para o consumo de álcool mesmo na ausência de fatores de risco. A maioria dos pacientes idosos atendidos na APS não são rastreados e muitos casos acabam sendo subnotificados. O uso de questionários mais curtos durante a triagem se apresenta como uma opção viável no atendimento do paciente idoso (Haighton et al., 2016; Shaker \& Ardnt, 2017).

\subsection{Eficácia da Intervenção Breve em Pacientes Idosos da Atenção Primária à Saúde}

A IB é um atendimento de curta duração sendo realizado após a triagem positiva para consumo de álcool. A IB busca modificar a conduta do paciente em relação a uso de álcool, auxiliando-o conscientizar-se sobre os riscos e malefícios à sua saúde, motivando-o a reduzir ou cessar seu consumo (Abreu et al., 2018; Branco et al., 2020). A implementação da IB em idosos tem demonstrado ser uma estratégia de sucesso, havendo reduções significativas no consumo e na frequência de uso de álcool, comprovando ser tão efetiva na população idosa, quanto em jovens. Em estudo realizado no Estados Unidos da América, idosos consumidores pesados de álcool apresentaram duas vezes mais chances de moderar seu consumo após a IB (Copeland et al., 2003; Pereira et al., 2013).

Segundo Copeland et al. (2003) a curto prazo, a IB permite que os idosos tomem consciência dos possíveis danos à saúde que o álcool possa ou já tenha causado, aumentando a capacidade de cuidarem de sua saúde. Já a longo prazo, a saúde do idoso tende a melhorar, levando a uma redução na necessidade de serviços de saúde. Borok et al. (2013) afirmam que idosos geralmente reduzem ou cessam o consumo de álcool quando são apresentadas evidências de que o álcool prejudica sua saúde e que a redução ou a abstinência irá beneficiá-los de alguma forma, motivando-os a adotar outros comportamentos e atitudes positivas em relação à saúde.

A IB pode ser aplicada por profissionais de saúde de várias formações que possuam capacitação e treinamento adequados. Salienta-se que a IB é indicada especialmente para pacientes com risco moderado e alto de consumo de álcool. Casos de dependência de álcool devem ser encaminhados para serviços especializados em dependência química. (Abreu et al., 2018; Branco et al., 2020). 


\subsection{Barreiras para Implementação de Triagem para Consumo de Álcool e Intervenção Breve}

Uma das barreiras para realização da triagem e aplicação de IB é a falta de tempo, geralmente os atendimentos são breves e há outras prioridades concorrentes. A ausência de espaço e ambiente adequados faz com que os pacientes se sintam desconfortáveis em relatar o consumo de álcool durante o atendimento, diante disso, profissionais da saúde acabam não investigando o uso por acreditarem que tais condições de trabalho sejam desrespeitosas ao atender o paciente. Além disso, o estigma somado a falta de conscientização e engajamento por parte dos profissionais de saúde, também os levam a não adotar estratégias de enfrentamento em sua rotina de trabalho. (Abreu et al., 2018; McNeely et al., 2018; Pereira et al., 2013).

Pacientes idosos podem se sentir descontáveis ao revelar o consumo de álcool por temerem uma reação negativa de quem os está atendendo ou não estarem prontos para discutir o problema. Idosos geralmente relatam sentimentos de vergonha e culpa por serem vistos como dependente químicos, acreditam ser tarde demais para mudança de comportamento e se beneficiarem do tratamento. (Haighton et al., 2016; McNeely et al., 2018; Soares et al., 2016)

É necessário que os pacientes acreditem que haverá benefícios decorrentes do tratamento, sendo imprescindível o estabelecimento de vínculo de confiança entre o profissional e o idoso. O profissional ao atender o idoso usuário de álcool deve adotar postura empática e respeitosa, compreendendo que o processo de envelhecimento está associado a mudanças físicas, psicológicas e sociais, tornado muitas vezes o idoso vulnerável ao consumo de álcool (McNeely et al., 2018).

Apesar de os profissionais de saúde serem treinados para abordar questões relacionadas à saúde mental, o consumo de álcool é menos investigado, havendo necessidade de melhor treinamento de profissionais da APS bem como investimento na educação permanente de toda a equipe de saúde, reforçando a realização de triagem universal e IB para consumo de álcool dos usuários do serviço, sobretudo para grupos menos investigados como os idosos (Zerbetto et al. 2017).

\section{Conclusões}

A pesquisa qualitativa possibilitou analisar as evidências literárias sobre as ações de prevenção e intervenção para o idoso dependente de álcool nos serviços de APS. Por meio da análise de conteúdo foi possível a estruturação dos dados obtidos nos seis artigos que compuseram essa Revisão Integrativa da Literatura, tornando o processo de categorização mais rigoroso, claro e bem delimitado. Os resultados mostraram que a APS desempenha um papel fundamental no atendimento aos idosos que apresentam quadro de consumo de álcool de risco e nocivo à saúde, ao implementarem ações de prevenção, identificação e tratamento, ressaltado que casos de dependência de álcool devem ser encaminhados para serviços especializados.

A implementação de tais ações, possuí eficácia e benefícios comprovados cientificamente na população idosa, porém pode-se afirmar que existem mais barreiras do que facilitadores para sua implementação enquanto rotina de trabalho.

O estigma associado a dependência de álcool faz com que os profissionais não se conscientizem a respeito da gravidade da doença. A falta de tempo e ambiente adequado também são barreiras que inviabilizam a execução de triagem e IB. É necessário maior engajamento dos profissionais da APS no atendimento de pacientes idosos consumidores de álcool, que geralmente não relatam seu consumo resultando na subnotificação de casos. 
É fundamental que os profissionais recebam treinamento e investimento em educação permanente, visando a implementação de triagem universal para consumo de álcool e IB. Diante da complexidade que envolve temática desta revisão integrativa, recomenda-se a realização de estudos visando a consolidação da eficácia da triagem para consumo de álcool e IB em idosos, tendo em vista a escassez literária nacional.

\section{Referências}

Abreu, A. M. M., Marinho, G. L., \& Jomar, R. T. (2017). Necessidade de intervenção breve por uso de álcool, tabaco e outras drogas entre usuários da atenção primária à saúde. Jornal Brasileiro de Psiquiatria, 66(2), 104-110. https://doi.org/10.1590/0047-2085000000157

Abreu, A. M. M., Jomar, R. T., Taets, G. G. C., Souza, M. H. N., \& Fernandes, D. B. (2018). Rastreamento e intervenção breve para uso de álcool e outras drogas. Revista Brasileira de Enfermagem, 71(Suppl 5). https://doi.org/10.1590/0034-7167-2017-0444

Adayonto, E. O., Adewole, A. J., \& Ehimigbai, M. (2019). Alcoholism amongest geriatric patients attending general practice clinic of a teaching hospital in Benin City, Nigeria. Nigerian Postgraduate Medical Journal, 26(4), 230-234. http://doi.org/10.4103/npmj.npmj_83_19

Borok, J., Galier, P., Dinolfo, M., Welgreen, S., Hioffing, M., Davis, J. W., Ramirez, K. D., Liao, D. H., Tang, L., Karno, M., Sacco, P., Lin, J. C., \& Moore, A. A. (2013). Why do older unhealthy drinkers decide to make changes or no in their alcohol consumption? Data from the healthy living as you age study. Journal of the American Geriatrics Society, 61(8), 1296-1302. https://doi.org/: 10.1111/jgs.12394

Branco, F. M. F. C., Ferreira, A. C. P. A., \& Diniz, T. M. M. A. B. (2020). Intervenções breves junto a utentes da atenção primária em uso de risco e nocivo de álcool. Cogitare Enfermagem, 25, e73502.: https://doi.org/10.5380/ce.v25i0.73502

Chagas, C., Tassiane, P., Machado, D, A., Martins, L. B., Opaleye, D., Piedade, T., Galduróz, J. C. Fernandes., \& Ferri, C. (2019). Alcohol consumption by older people in Brazil: A systematic review of population-based study. Addictive Disorders \& Their Treatment, 18(4), 229-237. http://doi.org/10.1097/ADT.0000000000000168

Copeland, L. A., Blow, F. C., \& Barry, K. L. (2003). Health care utilization by older alcohol-using veterans: Effects of a brief intervention to reduce at-risk drinking. Health Education \& Behavior, 30(3), 305-321. https://doi.org/:10.1177/1090198103030003006

Fink, A., Elliot, M. N., Tsai, M., \& Beck, J. C., (2005). An evaluation of an intervention to assist primary care physicians in screening in educating older patient who use alcohol. Journal of the American Geriatrics Society, 53(11), 1937-1943. https://doi.org/: 10.1111/j.15325415.2005.00476. x.

Finn, S. W., Hammarberg A., \& Andreasson S. (2018). Treatment for alcohol dependence in primary care compared to specialized outpatient treatment - A randomized controlled trial. Alcohol Alcohol, 1(53), 376-385. https://doi.org/: 10.1093/alcalc/agx126

Guidolin, B. L., Filho, I. G. S., Nogueira, E. L., Junior, F. P. R., \& Neto A, C. (2016). Padrões de uso de álcool em uma amostra de idosos no município de Porto Alegre, Brasil. Ciência \& Saúde Coletiva, 21(1), 27-35. https://doi.org/: 10.1590/1413-81232015211.10032015

Haighton, C., Wilson, G., Ling, J., MacCabe, K., Crosland, A., \& Kaner, E. (2016). A qualitative study provision for alcohol related health issues in mid to later life. PLOS One, 11(2), e0148601. https://doi.org/10.1371/journal.pone.0148601

McNeely, J., Kumar, P. C., Rieckmann, T., Sedlander, E., Farkas, S., Chollak, C., Kannry, J. L., Vega, A., Waite, E. A., Peccoralo, L. A., Rosenthal, R. N. McCarty D., \& Rotrosen. J. (2018). Barriers and facilitators affecting the implementation of substance use screening in primary care clinics: a qualitative study of patients, providers, and staff. Addiction Science \& Clinical Practice, 13(1), 8. https://doi.org/:10.1186/s13722-018-0110-8

Ministério da Saúde. (2003). A política do Ministério da Saúde para atenção integral a usuários de álcool e outras drogas. Secretaria Executiva, Coordenação Nacional de DST/AIDS. http://bvsms.saude.gov.br/bvs/publicacoes/politica_atencao_alcool_drogas.pdf 
Moher, D., Liberati, A., Tetzlaff, J., \& Altman, D. G. (2009). The Prisma Group. Preferred reporting reporting itens for systematic reviews and meta-analyses: The PRISMA statement. PLOS Medicine, 6(7), e1000097. https://doi.org/10.1371/journal.pmed.1000097

Pereira, M. O., Anginoni, B. M., Ferreira, N. C., Oliveira, M. A. Ferreira., Vargas, D., \& Colvero, L.A. (2013). Efetividade da intervenção breve para o uso abusivo de álcool na atenção primária: Revisão sistemática. Revista Brasileira de Enfermagem, 66(3), 420428. https://doi.org/10.1590/S0034-71672013000300018

Sahker, E., \& Ardnt, S. (2017). Alcohol use screening and intervention by american primary care providers. Internacional Journal of Drug Policy, 41, 29-33. https://doi.org/: 10.1016/j.drugpo.2016.11.013

Soares, M. S., Lima, E. D. R. P., Naegle, M. A., Silva, P. A. B., Santos, J. F. G., \& Silva, L., B. (2016). Consumo de álcool e qualidade de vida em idosos na saúde da família. Revista de Enfermagem do Centro Oeste Mineiro, 6(3), 2362-2376. doi: https://doi.org/10.19175/recom.v6i3.1184

Souza, M. T., Silva. M. D., \& Carvalho. R. (2010). Revisão integrativa: o que é e como fazer. Einstein, 8(1), 102-106. https://doi.org/10.1590/s1679-45082010rw1134.

The Joanna Briggs Institute. (2014). Joanna Briggs Institute Reviewrs' Manual: 2014 edition/supplement. The University of Adelaide. https://nursing.Isuhsc.edu/JBI/docs/ReviewersManuals/Economic.pdf

Zerbetto, S. R., Furino, V. O., \& Furino F. O. (2017). A Implementação da intervenção breve na atenção primária à saúde: Revisão Integrativa. Revista Atenção Primária à Saúde, 20(1), 107-117. https://doi.org/10.34019/1809-8363.2017.v20.15782 